\title{
Modeling of Lower Hybrid Antennas using the ALOHA code and comparisons with Tore Supra experiments
}

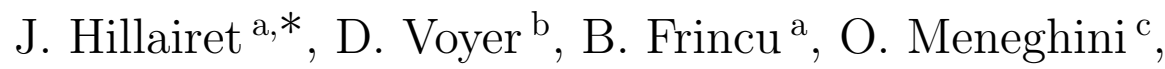 \\ A. Ekedahl ${ }^{a}$, M. Goniche $^{\text {a }}$ \\ ${ }^{a}$ CEA, IRFM, F-13108 Saint-Paul-lez-Durance, France. \\ ${ }^{\mathrm{b}}$ Centrale Lyon, 69134 Ecully, France. \\ ${ }^{\mathrm{c}}$ MIT Plasma Science and Fusion Center, Cambridge, USA.
}

\begin{abstract}
A new code for Lower Hybrid antenna design and cold plasma wave coupling, named ALOHA, is presented. ALOHA takes into account the poloidal dimension of the antennas and the coupling to slow and fast waves. Comparisons with experimental data for the LH antennas installed on Tore Supra and the TOPLHA code show that ALOHA is a fast and efficient predictive tool.
\end{abstract}

Key words: Lower Hybrid Current Drive (LHCD), predictive code, linear coupling theory, slow waves, fast waves, SWAN, ALOHA

\section{Introduction}

Over the last 30 years, slow waves in the lower hybrid (LH) range of frequencies have been used for various purposes. In particular, it was shown that the LH waves lead to a strong quasilinear Landau interaction with electrons, which can be used for non-inductive current drive (LHCD). Based on this properties, LHCD is commonly used to control the current profile and is a suitable candidate for sustaining steady state conditions in tokamak plasmas[1]. Experimentally, the LHCD showed its capacity to drive all the plasma current for 6 minutes in Tore Supra[2].

* Corresponding author.

Email address: julien.hillairet@cea.fr (J. Hillairet). 
Comprehensive modeling tools are required to interpret present day experiments and to design future LHCD antennas, such as the Passive-Active Multijunction (PAM) antenna which will be installed on Tore Supra in the framework of the CIMES project [3], as well as the LHCD system on ITER[4]. Since the creation of a new antenna design could be long and rely on an iterative process, the first development phase must use fast codes in order to avoid computer bottlenecks. Meaningful results in designing and optimizing microwave structures can be obtained from electromagnetic codes, in particular for vacuum parts of antennas such as mode converters or phase shifters. However, to the authors' knowledge, commercial codes cannot handle generic dielectric tensors, thus cannot account for fusion plasma coupling. Consequently, the LH antenna design process still requires adapted tools such as FELHS[5], GRILL3D[6] or TOPLHA[7].

Up to now, the design of LH antennas Mark I and Mark II in Tore Supra tokamak had been achieved with the help of the Slow Wave ANtenna (SWAN) code[8]. In SWAN, effects of coupling between a row of waveguides and the plasma edge are modeled relying on the classical two-dimensional linear theory of the Grill[9]. In this theory, the waveguide are assumed to extend infinitely in the poloidal direction, with perfectly conducting walls.

In order to improve this description, a new code named Advanced LOwer Hybrid Antenna (ALOHA) had been developed[10]. ALOHA is also based on the linear coupling theory and shares the same cold inhomogeneous plasma hypothesis as SWAN, but takes into account the poloidal waveguides finite size effects thus the fast waves on plasma coupling. This new code is a fast and efficient predictive tool to help designing LHCD antenna and modeling LH wave coupling to Tokamak plasmas. ALOHA takes part of the EFDA Integrated Tokamak Modeling (ITM) Taskforce IMP\#5, as input to ray tracing codes[11].

In section 2, a brief description of the ALOHA code is given, then in section 3, comparisons with Tore Supra experiments and the TOPLHA code are presented. Finally, as conclusion, some perspectives for the future development of ALOHA are presented.

\section{The ALOHA code}

In order to facilitate the analysis, the design process is split into two distinct parts: the antenna and the plasma descriptions. This organization allows to switch easily among antenna designs (number and size of waveguides, etc.) and plasma conditions (edge density profile, magnetic field amplitude, etc.). 
In ALOHA, an antenna is described on the toroidal and on the poloidal directions as a succession of modules. Each module contains a primary waveguide (input) and a phased array of narrow waveguides (ouput), as illustrated in fig. 1(a)). The waveguide walls are assumed perfectly conducting, the medium within is taken as free-space and the fields are described using modal expansion. The incident power and phase information for each module can be either taken from the experimental data or analytically defined.

In order to take into account refinements in the antenna design, such as the curved shape of the antenna front face which was previously neglected in SWAN, the ALOHA code allows to use the scattering matrix of each module. These matrices can be either taken from experimental measurements or imported from internal or commercial electromagnetic codes, such as Ansoft HFSS.

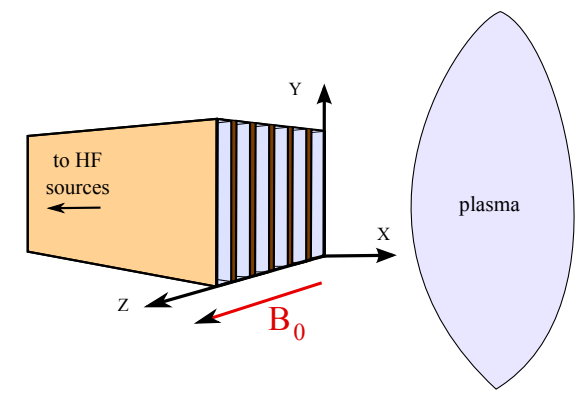

(a)

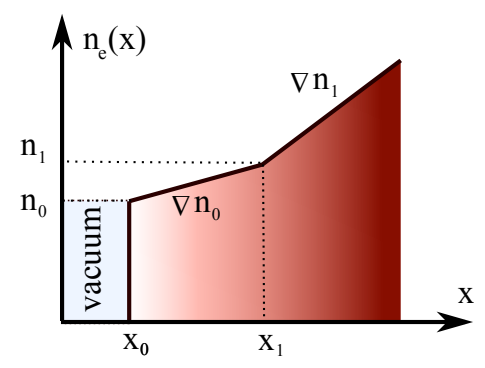

(b)

Fig. 1. 1(a): General geometry used in ALOHA. 1(b): General description of the edge plasma density profile in ALOHA: a step at $x=x_{0}$ then 2 linear density gradients.

The second part consists in the plasma description. Due to the fact that the electromagnetic fields scattered by the antenna is supposed to be absorbed beyond the coupling region, into the core plasma, only the edge density profile is of interest here. In ALOHA, this density profile is supposed to be homogeneous in $y$ and $z$ for each poloidal row. In the $x$ direction, which is perpendicular to the confining magnetic field (cf. figure 1(b)), the electron density can be defined with a density step, followed by one or two linear profiles. In addition, different density profiles can be assumed for different poloidal rows.

The ALOHA code computes the plasma coupling of all the waveguides, with an advanced implementation of the linear theory presented by BERS and THEILHABER[12]. Near the mouth of the antenna, the evanescent modes which can be excited are taken into account and it is assumed that the launcher is opened through a infinite perfect electric conducting surface. At the vacuum-plasma interface, the boundary conditions are expressed through the plasma surface admittance in the spectral domain. The ALOHA code implements $1 \mathrm{D}$ and $2 \mathrm{D}$ descriptions of the electromagnetic waves propagation into the plasma 
(ALOHA-1D and ALOHA-2D). The plasma surface admittance is generally a rank-2 tensor, which links the spectral tangential components of electric and magnetic fields at the interface. In a 1D propagation description, the admittance is then simply a scalar as in the classical linear theory of the Grill. A future communication will present in details the theory underlying the ALOHA code[13].

Finally, ALOHA computes the radiated spectrum, the directivity and the electric fields at the mouth of the antenna. The reflection coefficients in the primary waveguides are deduced from the scattering matrix and the incident power in each module. The reflection coefficients can be then compared with experimental data.

\section{Comparison of ALOHA with experimental data and the TOPLHA code}

\subsection{Comparison with experiments}

In this section, a comparison between experimental measurements on the Mark II LH antenna currently installed in Tore Supra and ALOHA-1D predictions are presented. In order to avoid possible non-linear effects, low power pulses were used, i.e. pulses for which the power density at the mouth of the launcher is less than $3 \mathrm{MW} / \mathrm{m}^{2}$ (corresponding to an input power of $500 \mathrm{~kW}$ ). The main plasma parameters are identical for the selected pulses. A variation in the density in front of the antenna (between $2 \cdot 10^{17}$ to $6 \cdot 10^{17} \mathrm{~m}^{-3}$ ) was obtained by varying the plasma position.

In fig. 2, the experimental reflection coefficients at different electron densities, measured during Tore Supra pulses \#39202, \#39283-39286 for the 4 first lower modules of the Mark II antenna are plotted. The density is measured with the nearest Langmuir probe placed at the bottom of the launcher, close to the module \#1. Two curves computed with ALOHA are superimposed on these plots. The plain line corresponds to two adjacent layers defined with two different density gradients and no vacuum layer $\left(x_{0}=0\right)$. Near the antenna, the first layer is defined by $n_{e} / \nabla n_{e 0}=2 \mathrm{~mm}$, then the second layer by $n_{e} / \nabla n_{e 1}=2 \mathrm{~cm}$. This choice is justified by the fact that the side limiters of the antenna protrude by $2 \mathrm{~mm}$ and consequently produce a short gradient (2 mm) near the antenna mouth[14]. The dashed line corresponds to edge plasma described with a single density decay length of $2 \mathrm{~cm}$, as in SWAN.

The plain curve agrees better with the reflection coefficients than the dashed curve, in particular for modules 3 and 4 located in the center of the launcher, 

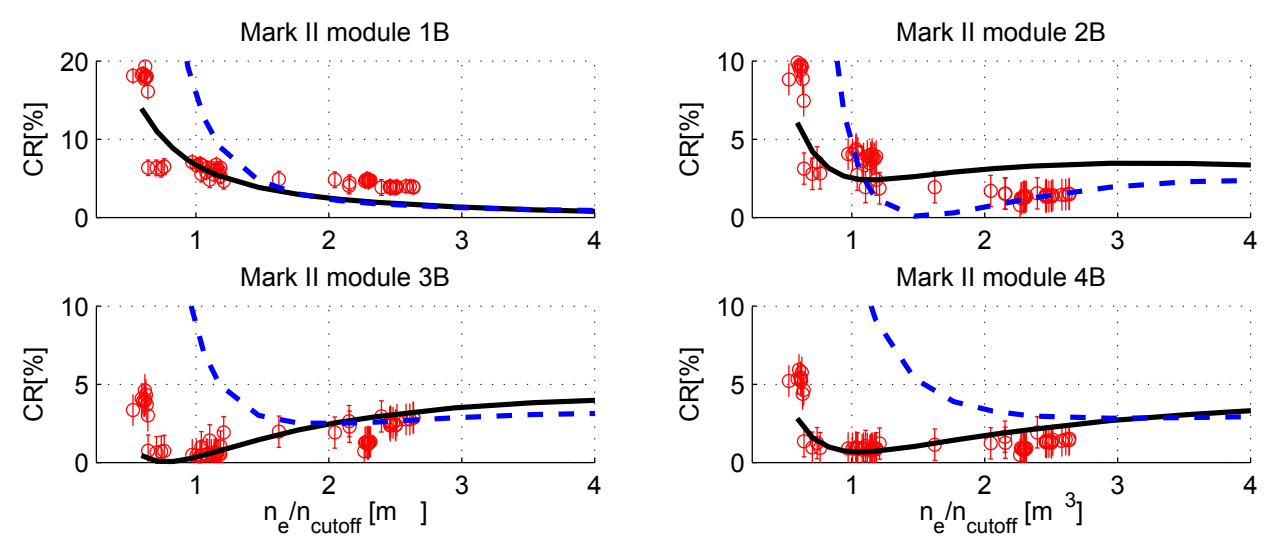

Fig. 2. Reflection coefficients on Mark II antenna versus ratio of electron density to cutoff density $n_{e} / n_{\text {cutoff }}$ for low power pulses on Tore Supra $\left(n_{\text {cutoff }}=1.71 \cdot 10^{17} \mathrm{~m}^{-3}\right)$. The plain curve represents the ALOHA-1D result, where the plasma is described with 2 density gradients. The dashed curve is for a single density gradient. The electron density is measured by the nearest Langmuir probe of the modules.

far from the side limiters. For low power pulses, extensive comparisons with ALOHA calculations indicate that the electron density is neither homogeneous in the toroidal direction, nor symmetric with respect to the center of the antenna. This phenomenon could be explained by a shadowing effect of the side limiters and the curvature of the magnetic field lines. However, it is worth noting that the absolute measured electron density is uncertain and a relative error of $1 \Delta 10^{17} \mathrm{~m}^{-3}$ may be taken in account in fig. 2 .

\subsection{Comparison with the TOPLHA code}

In order to validate ALOHA and to circumvent the incertitude of the edge density measurements, comparisons have been done with the TOPLHA code[7] on a simple geometry which consists of 8 adjacent waveguides (fig.3(a)). The waveguides are fed with different phasing at $4.6 \mathrm{GHz}$, on a plasma described with an edge density of $n_{e}=5 \Delta 10^{17} \mathrm{~m}^{-3}$ and a density gradient of $\nabla n_{e}=$ $1 \Delta 10^{20} \mathrm{~m}^{-4}$. The reflection coefficients for each waveguide are plotted in fig. 3(b) for various phasing. A good agreement between the two codes is found, although some differences exist for the $60^{\circ}$ phasing case. However, in this case, the $n_{\|}$spectrum of the structure is close to 1 , where the linear coupling theory ceases to be valid. A future communication will be dedicated on detailed comparisons between the two codes. 


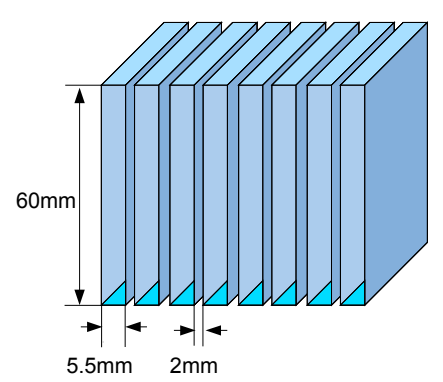

(a)

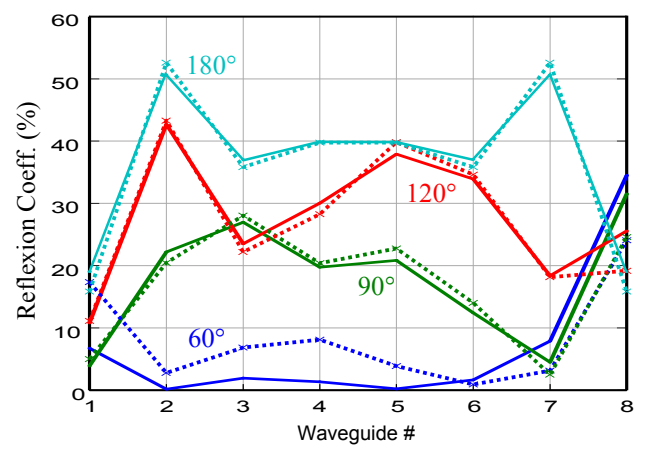

(b)

Fig. 3. Reflection coefficients comparison between TOPLHA and ALOHA for different phase differences between waveguides. 3(a): geometry of the 8 waveguides. 3(b): bold curves: TOPLHA; dashed curves: ALOHA.

\section{Conclusion and perspectives}

This paper has introduced ALOHA, a fast code for LH antenna coupling to cold inhomogeneous plasmas. This code is an evolution of the linear coupling code SWAN, and takes into account the poloidal size of the waveguides, advanced plasma descriptions near the edge of an antenna, thus the interaction of slow and fast waves between the waveguides and the plasma.

The validations of the code with Tore Supra experimental data and with the TOPLHA code show a good agreement. ALOHA is an efficient predictive tool, which is fast enough to be used during an iterative design process of a LHCD antenna. Moreover, the code is perfectly integrated to the conjoint use of commercial codes, which take part of the whole design process, in particular for vacuum parts of microwave structures.

ALOHA is presently evolving with many new options under development, such as generic plasma density profile in front of the waveguides. The other extensions of the code also under work are the coupling with ray tracing codes in the frame of the ITM/IMP\#5 taskforce, the account of hot plasma effects and electron heating in front of the launcher.

\section{Acknowledgements}

This work, supported by the European Communities under the contract of Association between EURATOM and CEA, was carried out within the framework of the European Fusion Development Agreement. The views and opinions expressed herein do not necessarily reflect those of the European Commission. 


\section{References}

[1] A.Tuccillo et al., Plasma Physics and Controlled Fusion 47 (2005) pp. B363B377.

[2] D.van Houtte et al., Nucl. Fusion 44 (2004) pp. L11-L15.

[3] J.Belo, Ph.Bibet et al., Fusion Eng. and Design 74 (2005) pp. 283-288.

[4] Ph.Bibet et al., Fusion Eng. and Design 74 (2005) pp. 419-423.

[5] R.Bilato and M.Brambilla, in 35th EPS Plasma Physics Conf., Greece (2008) pp. P5.094.

[6] M.A.Irzak and O.N.Shcherbinin, Nuclear Fusion 35 (1995) pp. 1341-1356.

[7] O.Meneghini et al. to be presented at APS 2008.

[8] D.Moreau, EUR-CEA-FC1246 Euratom-CEA (1984).

[9] M.Brambilla, Plasma Phys. 18 (9) (1976) pp. 669-674.

[10] S.Berio, Ph.Bibet,in 23rd EPS Conf. on Controlled Fusion and Plasma Physics (1996).

[11] A.Becoulet et al., Computer Physics Communications 177 (2007) pp. 55-59.

[12] A.Bers, K.Theilhaber, Nuclear Fusion 23 (1) (1983) pp. 41-44.

[13] D.Voyer, to appear in Nuclear Fusion.

[14] F.Leuterer et al., Plasma Phys. and Controlled Fusion 33 (3) (1991) pp. 169-180. 